\title{
SARS-CoV-2 on the move: Reviewing Innate vs Acquired for Covid-19
}

\author{
Sangam Banerjee* \\ Saha Institute of Nuclear Physics, 1/AF, Bidhannagar, Kolkata, 700064 India \\ email: sangam.banerjee@saha.ac.in
}

\begin{abstract}
:
In this article we have reviewed and presented certain facts published so far and have raised some pertinent questions to understand the progress of Covid-19 disease. We have discussed the important role of innate and acquired immunity in Covid-19 disease. We have discussed why immunity fails leading to 'immunity escape'. We have tried to address few concerns such as: Can vaccines drive the pathogen to mutate to a higher virulent strain? Can this lead to new variants and eventually leads to evade immunity? Demand for new design of vaccine such as antibody cocktail. Effect of imperfect leaky vaccine. We have also discussed the challenges of antigenic drift, antigenic shift and the role of antigenic sin which determines the scope of cross-reactivity with other variants from Covid-19 vaccine
\end{abstract}

\section{Introduction: How Covid-19 progressed since January 2020?}

The coronavirus disease-19 (COVID-19) is caused by the severe acute respiratory syndrome coronavirus 2 (SARS-CoV-2). When covid-19 disease was progressing around the world from the beginning of 2020, it was found that in certain countries the progression of the disease was relatively much slower than many others (see fig. 1). The slow progression of the disease could be attributed to the presence of better innate immunity and this resulted in flattening of the disease progression curve along with shifting of the peak to later time if at all to achieve herd immunity threshold (HIT) value as shown in fig. 1. The flattening and delay in the peak will depend on the strength of innate immunity of the population. Subsequently immediately it was observed that in those countries where the rise in the disease was initially sharp, by May 2020 it was on the decline. By then, preventive measures such as lockdown, social distancing, face mask etc., were imposed and practiced by almost all countries world-wide. However, the countries where the progression of the disease was very slow, were yet to attain the peak in the disease progression (see fig 1(b)). The question was: - If herd immunity is to be achieved by every country, then the countries having slow progression are far behind and will take much longer time to reach the HIT value. It seemed, that this group (Group B, Fig.1(b)) were already protected by certain immunity resulting in the flattening of the disease progression curve.

But, interestingly none of the country which were showing decline in their disease progression curve had achieved HIT, but the pandemic seemed to be coming to an end in a matter of days. However, interestingly we saw that some countries were experiencing a second wave (some even third wave) of this disease. The countries where the progression of the disease (in 2020) was steeper were having the second wave earlier than the countries where the progression of the disease was slower, which is very clear from Fig. 2 . In some countries where strong preventive measures

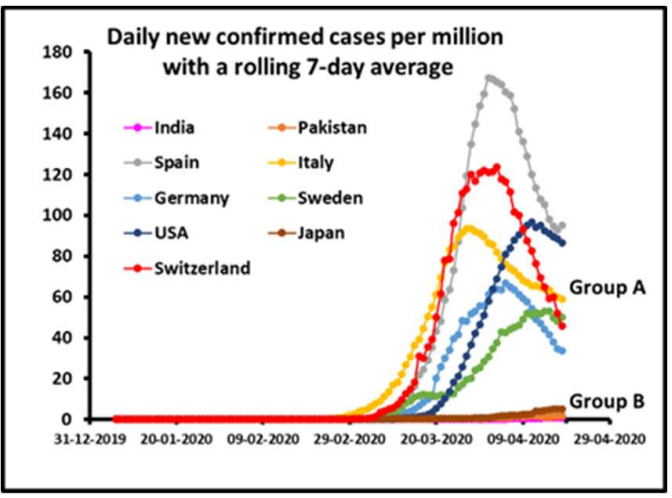

(a)

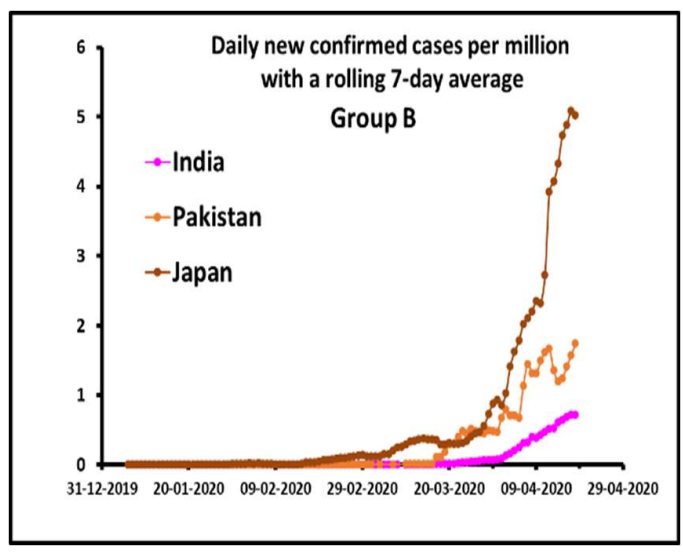

(b)

Fig 1. (a) and (b): Daily new confirmed cases per million with a rolling 7-day average of different countries with data acquired from https://ourworldindata.org/coronavirus shown up to 13th April 2020. Group B shows slow progression of the disease than Group A. 
were taken or large-scale mass-vaccination are being undertaken the disease progression shows plateauing instead of going down to zero. This is now a very serious and dangerous turn of an event and we need to understand this phenomenon and look into it in detail.

(a)
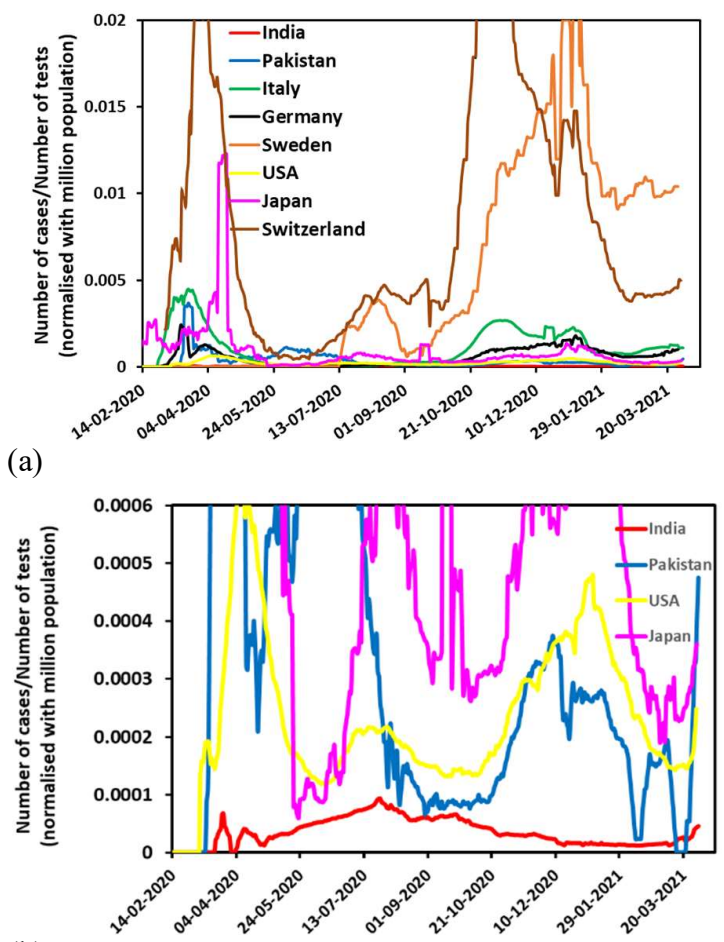

(b)

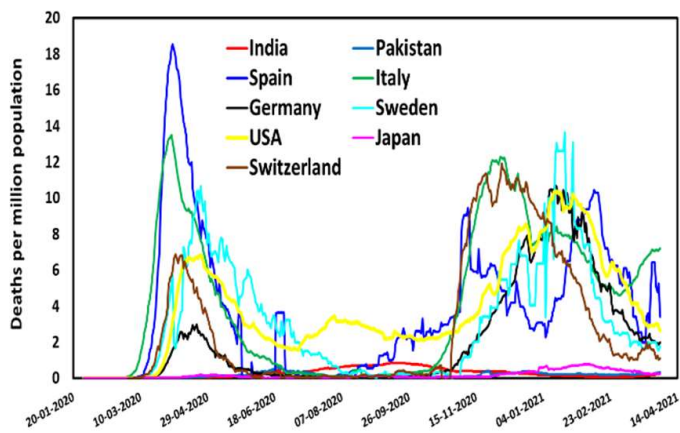

(c)

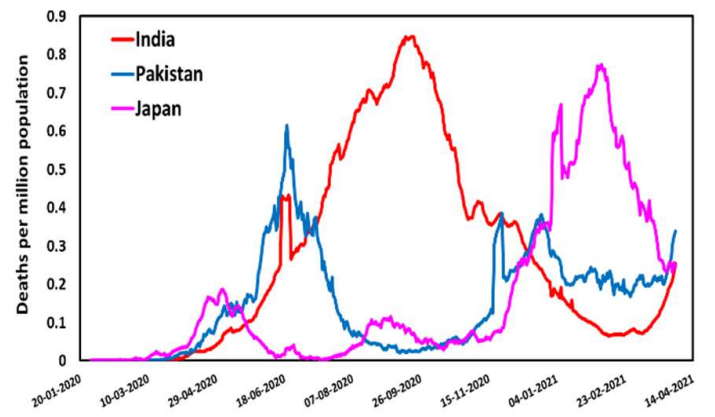

(d)

Fig. 2. (a) and (b): Daily new cases per number of tests per million population with a rolling 7-day average of different countries with data acquired from https://ourworldindata.org (c) and (d) death per million population

To understand this, we present in this manuscript (briefly) the science behind the infection and rethink the strategy of curbing the disease. In order to do this, we have to understand briefly the mechanism of how our body's immune system wins over the virus or any other pathogens per say.

In this article we shall discuss the following points sequentially:

1. Understanding innate immunity in brief.

2. Understanding adaptive immunity in brief.

3. Why immunity fails leading to immunity escape?

4. Can vaccines drive the pathogen to mutate to a higher virulent strain?

5. Evolution of more virulent pathogens.

6. Evidence of new variants.

7. Simulation evidence of variants evading immunity.

8. Antibody cocktail.

9. SARS-CoV-2 evades $\mathrm{T}$ cell responses - an evidence

10. Imperfect leaky vaccine.

11. Complete Mapping of Mutations to the SARSCoV-2 Spike Receptor-Binding Domain.

12. Where is the originally identified SARS-CoV-2 (labeled as D614) found first in Wuhan, China in Dec 2019.

13. Deletion of spike glycoprotein itself: How to fix the missing?

14. What is the present status?

15. To be or not to be vaccinated?

16. A very common question 'Is natural infection better than immunization?

17. The challenge of antigenic drift and antigenic shift.

18. What causes variant and can it be controlled?

19. A new dawn in the fight against the disease: to be or not to be worried?

20. Judging the risk "risk management".

21. Prophylactic measures for covid 19: Changing Diet and life style.

22. Maneuvering the pandemic.

23. Where are we now: A birds eye view

24. Remarks - Take away message.

25. Conclusion.

\section{Understanding innate immunity in brief:}

To understand our self-defense mechanism from pathogens, we have to first understand our immune system. Immunity can be defined as a complex biological system able to recognize and tolerate what belongs to our self and to reject what is foreign (nonself). Our immune system can mainly be classified into two categories - innate and adaptive. The innate immune defense system is the first line of defense against the invading pathogens. These immune cells are originated in the bone marrow and then migrate to skin, gastrointestinal tract, respiratory tract, 
nasopharynx, cilia, eyelashes and other body hair and lymphatic system where it circulates in the blood. It is also present in secretions such as mucous, bile, gastric acid, saliva, tears, and sweat. The innate cells comprise of the leukocytes and phagocytes. Leukocytes secrete chemicals as an early warning signal immediately as soon as pathogens are detected (termed as chemotactic factor along with complement protein secreted by our infected cell resulting to anti-coagulant and vasodilation so that these molecules can move freely). The job of destroying the pathogen is accomplished by the phagocytic cells which gets attracted to the pathogens on getting the signal in this process. The phagocytic cell simply surrounds the pathogen and engulf them and the enzymes in the cell break down the pathogen and destroys it. The names of the members of leukocytes are natural killer cells, mast cells (in tissue), eosinophils (in blood) and basophils (in blood) and the names of the members of phagocytic cells are macrophages, neutrophils and dendritic cells. To understand further is to know how our immune system distinguish pathogens from self-components to selectively destroy only the pathogen. This is achieved by the innate immune system, which through the evolutionary process have learned to detect evolutionarily conserved (non-changing pattern) signatures from pathogens having a wide variety of patterns of relatively invariable receptors on its surface (pathogen associated molecular patterns, PAMPs). Innate immune system recognizes PAMPs by the pattern recognition receptors (PRRs) to distinguish pathogens from self-components (An analogy - we identify any object with its pattern, in molecular level any chemical reaction can also be considered as pattern recognition for the reaction, only certain pattern key will fit certain pattern lock). Engagement of PRRs leads to the activation of some of these cells and their secretion of cytokines and chemokines, as well as maturation and migration of other cells (adaptive/acquired self-defense gets activated). If the innate immunity is not fast and efficient enough to neutralize the pathogens compared to multiplication of the pathogen, it then creates an inflammatory environment that leads to the establishment of the adaptive immune response which renders further help and joins the fight.

\section{Understanding adaptive immunity in brief:}

If the innate immune response is insufficient to control an infection, then the second line of defense is activated i.e., the adaptive (acquired) immune system composed of specialized cells and molecules that eliminate pathogens or prevent their growth and this is termed as adaptive immune response which is found in vertebrates only. This adaptive response develops slowly (over days to weeks), and are 'precisely' targeted immune responses. Some of the innate immunity cells (from the innate immunity branch) such as dendritic cells, macrophages, Langerhans cells and $\mathrm{B}$ cells after processing (fragmenting) the pathogens prepares itself for presenting antigens (an antigen here is the portion of the pathogen that causes your immune system to produce antibodies against it) for recognition by lymphocytes such as $\mathrm{T}$ cells. Thus, the innate immune cells also act as antigen-presenting cell (APC) that displays antigen complexed with a protein called major histocompatibility complexes (MHCs are proteins on the cell surface involved in antigen presentation to $\mathrm{T}$ cells) on their surfaces. T cells have affinity towards MHC complexes and recognizes these complexes as their self and also the pathogen fragments (i.e., antigens as foreign) presented using their $\mathrm{T}$ cell receptors (TCRs). T cells are found in the blood and are also concentrated in central lymphoid organs and tissues, such as the spleen, tonsils and lymph nodes. Now at this stage the initial adaptive immune response is triggered. As soon as antigen complexed with MHC is recognized by the $\mathrm{T}$ cells it becomes activated and once activated, they divide rapidly and migrates to inflammation zone containing infected cell sites destroying infected cell by cytolysis or by apoptosis. To cut the story short, in this process T cells along with $B$ cells tries to finish the unfinished job of innate immunity. As an additional information, $\mathrm{T}$ cells are produced in bone marrow and matures in the thymus and hence are called as $\mathrm{T}$ cells and are programmed to be specific for one particular foreign particle (antigen). Once they leave the thymus, they circulate throughout the body until they recognize their antigen on the surface of APCs. Whereas, B cells are produced and matured in bone marrow, goes to infected site and releases antibodies which binds to antigens on the pathogen surface and thus making the pathogen immobile and harmless until the phagocytes destroy it. It is to be noted that activated adaptive immune cells further drive innate cells to amplify anti pathogen responses. Some T cells (called as Th1) activate macrophages and some $\mathrm{T}$ cells (called as Th2) activate eosinophils through cytokine release and $\mathrm{B}$ cells secrete antibodies to activate the cascade of complement proteins, phagocytes, natural killer (NK) cells and mast cells. Thus, the two arms of the immune system (innate and adaptive) and the mutual amplification of responses helps effectively and efficiently to combat microbial pathogenic invaders. In brief, the innate immune response is able to slow down viral replication and activate cytokines which trigger the synthesis of antiviral proteins. The adaptive immune system neutralizes virus particles and destroys infected cells. With this sufficient background and by knowing the complexity of our immune system, we now address how this very advance complex system fails to combat the simple invader and what then is the next step.

\section{Why immunity fails - immunity escape?}

The immune system not working properly can be the result of genetic deficiencies from the birth, suppression of immunity by medication and life style. Even over response immunity is as bad, such as 
allergies and immune response to one's self which is called as autoimmunity. These individuals are at higher risk of experiencing frequent infections and severe symptoms. We have now two options to adopt: (1) therapeutic drug and (2) prophylactic vaccines.

Drugs tend to target pathogens in a single time process whereas vaccines tend to target pathogens by inducing host-specific antibody and/or T cell responses remembering the pathogen for certain period of time or even for a life time. The pathogen can evade drug by simple mutation and become specific drug resistance (such as antibiotic resistance) whereas it is understood that more mutations are needed to become vaccine resistance (new antigenic strains). It is believed that vaccines stimulate the production of the right antibodies and boost the immune system to fight the pathogen if exposed to it in the future. Drug resistance is well studied and understood widely but what about vaccine resistance? What are the health consequences if the disease (pathogen) evolves to become vaccine resistance?

Vaccine resistance was first suspected when variation in key antigens and outbreaks in vaccinated populations were observed. Drug resistance pathogen can be handled by swapping drugs at the time of treatment but protection against a vaccine-resistant pathogen may require large scale campaigns at the population level. Whereas, vaccine resistance is not just about immune evasion (new variant or completely new antigenic strain occurring by genetic mutation); it can involve other phenotypes like immune suppression and faster replication to outrun vaccine-induced immunity [1-3]. Some of these phenotypes (the new variant or new strain) could cause more severe disease in unvaccinated individuals $[4,5]$. This sort of short coming can be avoided in animal breeding farms where every animal can be vaccinated at one go in a very short time, however, this large-scale vaccination in human population is not possible. Thus, vaccine resistance would be a great concern in human population. Immune memory can protect hosts from reinfection, but incomplete vaccine that allow future reinfection may inadvertently select more-harmful pathogens. We shall discuss this in more detail below.

\section{Can vaccines drive the pathogen to mutate to a higher virulent strain?}

So, an important question is - Can some vaccines drive the pathogen to mutate to a higher virulent strain due to survival pressure? Conventional wisdom is that the natural selection will remove highly lethal pathogens because if host dies this will greatly reduce the transmission. But vaccines that keep hosts alive but still allow transmission, the virus can evolve to very efficient and virulent strains which can circulate in a population [6]. It has also been pointed out that an imperfect protection due to the imperfect nature of host immune memory (adaptive or innate responses) can readily evolve the targeted pathogen due to selection pressure for higher virulence [3,7-8]. Despite the small subset of pathogens that grants complete and lifelong immunity following infection but imperfect immunity can cause reinfection and examples of these are widespread in humans [9-12] and other animals [1316]. Infectious agents can rapidly evolve in response to health interventions and hence the fitness advantage demands higher virulent strain. It is a Catch-22 that the same immune system that evolved to protect the host from infection can drive the pathogen to evolve to be more harmful for the case of imperfect immunity/protection.

\section{Evolution of more virulent pathogens:}

Andrew F. Read et. al. [1] have raised a question- "Whether pathogen adaptation to vaccinated hosts can result in the evolution of more virulent pathogens?" They argued that even though vaccines reduce the disease but if vaccines do not prevent infection, replication and transmission then it may allow more virulent strains to evolve and circulate (they termed it as "imperfect" vaccines). Natural selection will even favor their circulation if virulent strains have a higher transmission in the absence of host death or are better able to overcome host immunity. This has been demonstrated by Andrew F. Read et. al. while experimenting with Marek's disease virus (MDV), a highly contagious oncogenic herpes virus showing that vaccination elevate the fitness of highly virulent strains above that of less virulent strains if the vaccine is imperfect. Thus, life-saving vaccines have the potential to increase disease virulence in unvaccinated host population $[4,7,17]$. Transmissionblocking vaccines (sterilizing vaccine such as smallpox vaccine) do not allow evolution of pathogens to occur [1].

\section{Evidence of new variants:}

It is well known that the virus during a pandemic might be changing as it passes from person to person as is observed in the case of HIV where chance mutations in HIV help it to evade the immune system. Even the coronavirus (SARS-CoV-2) genetic sequences might have changed via mutations resulting in the change in the property of the virus as it traverses around the world. We know viruses are highly adaptable by invoking random genetic changes in their viral code. This endless evolutionary survival pressure of any pathogen causes the immune system to develop tactics to fight pathogens and in turn the pathogens endlessly develop tactics to evade it. Though a majority of these mutations do not significantly alter the behavior of the virus. But by some chance, a virus will strike upon a favorable mutation that helps improving its chance of survival. Recently Kevin R. McCarthy et. al [18] reported a new variant of the SARS-CoV-2 virus which selectively deleted a small bit of its genetic sequence code causing modification of the spike protein which helps the virus particles to penetrate cells. This modification of the section of spike protein 
disallows the antibodies to latch onto it and this minor change could help the virus to evade the immune system.

\section{Simulation evidence of variants evading immunity:}

More studies have been reported on mutation of SARS-CoV-2 to evade the immune system (antibodies). Here innate immunity is not the only main concern of discussion but acquired immunity via adaptive/vaccination is our concern too i.e., the efficiency of antibodies binding with the antigens for neutralizing the pathogen.

Recently, Shang and Axelsen [19] reported a study titled "The Potential for SARS-CoV-2 to Evade Both Natural and Vaccine-induced Immunity". Since it is well known now that SARS-CoV-2 attaches to the surface of susceptible cells through interactions between the receptor binding domain (RBD) of its spike protein and angiotensin converting enzyme type 2 (ACE2) anchored in cell membranes. They [19] investigated by computer modelling using solvent accessible surface area (SASA) calculation method whether the naturally occurring mutations in the spike protein are able to prevent antibody binding, yet while maintaining the ability to bind ACE2 causing viral infectivity. They tried to address some very pertaining questions related to infectious SARS-CoV-2 variants. They found (1) mutations involved in the portions of the spike protein that binds with ACE2 were functionally conserved so as to not disrupt the RBD: ACE2 binding, (2) they suggested that mutated forms of SARS-CoV-2 may be able to reinfect people who have recovered from an earlier infection. We should note here that since natural infection will tend to generate multiple antibodies to distinct aspects of the spike protein (distinct aspects/portion of the spike proteins are epitopes: epitope is the specific piece of the antigen to which an antibody binds). So since, SARS-CoV-2 has a relatively low genetic diversity and hence the immune evasion by mutation becomes increasing unlikely as the number of independent neutralizing antibodies increases in the case of natural infection. This is a ray of hope for us.

\section{Antibody cocktail:}

Several mutants have been identified in different continents, presumably in the absence of evolutionary pressure. When evolutionary pressure is applied, we must expect the potential for immune evasion to increase. Vaccines containing only one variant of the spike protein as an immunogen may induce little or no protection against variant strains. To have long-term efficacy against SARS-CoV-2 it may be necessary for vaccines to include multiple variants of the spike protein as immunogens (antibody cocktail [20-22]). This strategy is routinely applied against influenza where it is possible to predict which strains will become epidemic, and the antigenic features of those strains tend to be stable for a season. SARS-CoV-
2 differs since many variant strains are already circulating and their antigenic features are not stable.

\section{SARS-CoV-2 evades $T$ cell responses - an evidence:}

$\mathrm{CD} 8+$ is the glycoprotein in $\mathrm{T}$ cell receptor (TCR) and antigens inside a cell are bound to MHC-1 molecules and brought to the surface of the infected cell by the class MHC-1 molecule, where they can be recognized by the T cell. If the TCR is specific for that antigen, it binds to the complex of MHC-1 molecule and the antigen and then the T cell destroys the infected cell. Benedikt Agerer et. al. [23] reported a study titled "SARS-CoV-2 mutations in MHC-I-restricted epitopes evade CD8+ T cell responses" Their findings highlighted the capacity of SARS-CoV-2 to subvert $\mathrm{CD} 8+\mathrm{T}$ cell surveillance through point mutations in MHC-I-restricted viral epitopes. This leads to immunity escape from $\mathrm{T}$ cell.

\section{Imperfect leaky vaccine:}

It is believed that immune memory has evolved to protect hosts from reinfection, but incomplete responses that allow future reinfection may inadvertently select for more-harmful pathogens as has been pointed out by Fleming-Davies [6] in a report titled "Incomplete host immunity favors the evolution of virulence in an emergent pathogen" and similarly by Andrew F. Read et. al [1] in a report titled "Imperfect Vaccination Can Enhance the Transmission of Highly Virulent Pathogens". This idea was initiated by Sylvain Gandon et. al. [4] in a report titled "Imperfect vaccines and the evolution of pathogen virulence" and subsequently by M.J. Mackinnon et. al. [9] in an article title "Virulence evolution in response to vaccination: The case of malaria" and by Yiska Weisblum et. al [24] in a report titled "Escape from neutralizing antibodies by SARS-CoV-2 spike protein variants".

\section{Complete Mapping of Mutations to the SARS- CoV-2 Spike Receptor-Binding Domain:}

Recently Allison J. Greaney et. al. in their report [25] titled "Complete Mapping of Mutations to the SARS-CoV-2 Spike Receptor-Binding Domain that Escape Antibody Recognition" where they have shown the complete escape maps which can predict which mutations are selected during viral growth in the presence of single antibodies. They further enable the design of escape-resistant antibody cocktailsincluding cocktails of antibodies that compete for binding to the same RBD (Receptor-Binding Domain) surface but have different escape mutations. Therefore, complete escape-mutation maps enable rational design of antibodies and assessment of the antigenic consequences of viral evolution.

\section{Where is the originally identified SARS-CoV-2 (labeled as D614) found first in Wuhan, China in Dec 2019}


In a featured article in Nature magazine "Making sense of coronavirus mutations", Ewen Callaway [26] says that different SARS-CoV-2 strains haven't yet had a major impact on the course of the pandemic - but they might in future. Citing works of Korber, Montefiori [27] and others, they have warned that "G614 is increasing in frequency at an alarming rate" (the wild variety - the original virus which originated in Wuhan, China was labeled as D614). G614 has rapidly become the dominant SARS-CoV-2 lineage in Europe and had then taken hold in the United States, Canada and Australia. G614 represented a "more transmissible form of SARS-CoV-2", one that had emerged as a product of natural selection. Functional importance of the G614 mutation in the SARS-CoV-2 spike protein has been discussed in detail by Cody B. Jackson et. al. [28] and pointed out reasons to be hopeful because they claim that there does not appear to be major selective pressure on the spike (S) glycoprotein from the antibody response, likely because the virus moves to a new host quickly before a neutralizing antibody response can develop. Korber et al. [27] presented evidence that there are now more SARS-CoV-2 viruses circulating in the human population globally that have the G614 form of the spike protein than the D614 form that was originally identified from the first human cases in Wuhan, China. Follow-up studies show that patients infected with G614 shed more viral nucleic acid compared with those with D614 and G614-bearing viruses show significantly higher infectious titers in-vitro than their D614 counterparts.

\section{Deletion of spike glycoprotein itself: How to fix missing?}

Most recently, Kevin R. McCarthy et. al [18] have pointed out in article titled "Recurrent deletions in the SARS-CoV-2 spike glycoprotein drive antibody escape", that they find recurrent deletion regions (RDRs) in the spike glycoprotein. These RDRs may pose resistance to neutralizing antibodies. These deletions appear to accelerate SARS-CoV-2 antigenic evolution.

\section{What is the present status?}

By now we are aware about the appearances of new variant and immunity escape by SARS-CoV-2. In fig. 2 we have plotted the disease progression of the same countries as plotted in fig. 1 and compared the disease progression normalized with testing numbers. Number of testing has increased in all the countries compared to one year ago. We clearly see the increase in the slope and magnitude in the rise of number of positive cases recently in many countries compared to last year increase and this indicates the virus is spreading at higher rate than it was earlier a year ago.

\section{To be or not to be vaccinated?}

To answer this, we have to still understand some more basics regarding virus behavior and its life cycle. We now know that virus while multiplying and making its copies make errors and this we call as mutation. Minor modification during making copies is called a variant and if the error accumulates then the virus becomes a new virus and we call it as antigenic shift. The new variant can reinfect and if the virus remains for too long then the immune system becomes hyperactive which leads to cytokine storm (in cytokine storm the host immune response becomes hyperactive, inflammatory response flaring out of control leading to excessive damage of the host cell).

Prophylactic vaccines come to rescue in this scenario where these are designed to build immunity in a patient. Vaccination involves introduction of antigens into a person's body with the aim that immune system will create antibodies for those antigens and be ready to fight the invader as soon as it invades. It is interesting that our immune system remembers and memorizes the antigen (invader) where $\mathrm{B}$ and $\mathrm{T}$ cells create memory cells. These clones of memory cells remain in the body, having information about each antigen the body has been exposed to. This gives our immune system the memory for recognizing the threat and jump into action with the army of antibodies to neutralize the threat when required. How long this memory last depends on the disease and this aspect of longevity of immunological memory is still not understood.

Can these memories be useful in the case of a variant attack? It seems that this memory is not very effective- as recently been reported in news article in Science magazine with heading "Vaccine-makers ponder how to adapt to virus variants", by Kai Kupferschmidt [29]. The variants which are talked about now are B.1.1.7 (first detected in the UK), B.1.351 (first detected in South Africa), and P.1 (detected in Manaus, Brazil). Other variants have also emerged, the mutation have allowed the virus to escape immune response. Vaccines developed against the original virus have also been found to be less effective against B.1.351.

A news analysis in BMJ "Covid-19: Where are we on vaccines and variants?" by Elisabeth Mahase [30], discuss this issue in some details. Published in Nature, Ewen Callaway \& Heidi Ledford [31] wrote an article titled "How to redesign COVID vaccines so they protect against variants - Lineages that can evade immunity are spurring vaccine makers to explore ways to redesign their shots". Here they say "Researchers are still debating whether the new variants could undercut the effectiveness of these first-generation COVID-19 vaccines. But some vaccine developers are moving forward with plans to update their shots so that they could better target the emerging variants, such as those identified in South Africa and Brazil. These lineages carry mutations seems to dampen the effects of antibodies which is crucial to fending off the infection. Researchers are also considering the possibility that 
vaccines against the coronavirus might have to be updated periodically, as they are for influenza" [31].

Now knowing that virus can evade immune system the question still remains regarding effectiveness of the vaccination against the variants. This question has been addressed by Wilfredo $\mathrm{F}$. Garcia-Beltran et. al in a recent article [32] titled "Multiple SARS-CoV-2 variants escape neutralization by vaccine-induced humoral immunity", they summarized their studies stating that "viral evolution will continue and it is possible that current vaccines will still provide clinical benefit against variants that exhibit poor cross-neutralization, such as P.1 and B.1.351, by reducing COVID-19 disease severity, but this has yet to be determined. Ultimately, it will be important to develop interventions capable of preventing transmission of diverse SARS-CoV-2 variants, including vaccine boosters that target these variants or technologies capable of eliciting or delivering broadly neutralizing antibodies" [32].

\section{A very common question 'Is natural infection better than immunization?'}

To answer this, we know that infected by chickenpox may lead to pneumonia, from Haemophilus influenzae type b (Hib) may lead to intellectual disability, pneumonia from pneumococcus, birth defects from rubella, liver cancer from hepatitis B virus or death from measles. Whereas, unlike natural infection, immunization does not extract such a high price for immunity; that is, immunization does not cause pneumonia, intellectual disability, birth defects, cancer or death. In some cases, vaccines induce a better immune response than natural infection: such as human papillomavirus (HPV) vaccine, tetanus vaccine, haemophilus influenzae type $b$ (Hib) vaccine, pneumococcal vaccine and some more. Thus, vaccines provide us protection with lesser quantities of deactivated virus or bacteria. The development of safe and efficacious vaccination against diseases that cause substantial morbidity and mortality has been one of the foremost scientific advances of the 21 st century.

\section{The challenge of antigenic drift and antigenic} shift:

It is well known that the regular recurrence of influenza epidemics is caused by antigenic drift to allow influenza to reinfect the same host. Vaccine mismatch can impact on vaccine effectiveness. To meet the challenge of antigenic drift, vaccines that grant broad protection against variant strains are needed against seasonal, epidemic and pandemic infection. Development of a universal vaccine and the use of vaccines that exploit mechanisms of crossprotective immunity is required.

Antigenic drift is the gradual evolution of viral strains due to frequent mutations. It occurs with time in response to selection pressure to evade human immunity. The process of antigenic drift is subtle, involving point mutations within antigen sites such that the binding of host antibodies is affected. Thus, allowing the virus to spread more rapidly among the population. Whereas, antigenic shift results into a new virus that have never been present in human circulation. This can have a significant impact causing new pandemics or epidemics.

\section{What causes variant and can it be controlled?}

By now we have got some hint regarding the mutation occurring with time in response to selection pressure resulting into evading immunity. These mutations are common in RNA viruses as that is their nature because RNA molecules are unstable and during copying it does not have a built-in proofreading (error correction) in their replication step and hence make mistakes unlike the DNA viruses (such as herpes, smallpox, and human papillomavirus (HPV)) where the host cell can often correct the mistakes. Hence mistakes in copying RNA happens without correction (variants) which means mutations accumulate rapidly as the virus multiplies in one person and if these mistakes are advantageous then it can have grave consequences for their hosts. The rapid rate of advantageous evolution can quickly develop resistance to anti-pathogen drugs and vaccines. But in some cases, mutations may actually lead to a weaker virus where the antigenic changes are so slight that there is no noticeable difference even in the disease's transmission and fatality rates. So, we have to understand what decide this factor?

If the virus variants make them more easily transmittable (strong antigen-ACE receptor binding) from one person to another leading to a quick virus spread and if it is less deadly causing less severe disease then it is better for the population to reach HIT value as quick as possible. If the new virus variants are deadlier, cause more severe disease and make COVID19 vaccines ineffective, then it is of great concern to humanity.

It is to be noted that when two variants infect (coinfection) the same cell, they can recombine, which is different than re-assortment (mutation). In recombination, a new single RNA genome is stitched together from pieces of the two "parental" viruses' genomes. This happens rarely and the resulting virus is termed as "novel virus." There is other mechanism such as virus jumping from humans to other animals and jumping back to humans. The probability of both the mechanism as well as re-assorting increases as the disease lingers in the population for longer time.

The SARS-CoV-2 variants found in the UK, South Africa and Brazil - more properly referred to as the B.1.1.7, B.1.135 and P.1 variants - are examples. All are reported to have significantly higher transmission rates than earlier lineages. Mutations causing two amino acid changes in the spike (S) protein of the B.1.135 variant, cause the virus to bind significantly more efficiently to the human ACE2 
receptors. This means that this and similar variants can spread more rapidly and efficiently within the human host population.

The initial variant (first found in Wuhan China) caused infection in the respiratory tract. But the new variant due to strong binding SARS-CoV-2 with ACE 2 means it can also infect and damage intestinal and urogenital tissues, cells of the central nervous system, and the endothelial cells of minor blood vessels, leading to clotting complaints and even strokes.

Above we discussed the complexity of the immunity system. Natural immunity leads to broad antibody and cellular immune responses that target many parts of the virus. But most SARS-CoV-2 vaccines stimulate responses that target only the $\mathrm{S}$ protein, and this has led to concern that new variants may escape these "narrow" immune responses. As discussed above that there are already several variants of SARS-CoV-2 detected by genomic surveillance. We also know that the $S$ proteins of seasonal common coldcausing coronaviruses evolve to avoid host immune responses, just as influenza viruses do, which results in people falling prey to these individual viruses every three years or so. This means that SARS-CoV-2 vaccines may have to be changed regularly, just as flu vaccines are. If mutation increases the pathogenicity of the virus along with transmissibility, then it is a deadly combination, but fortunately this has never been observed in the history for the case of human common cold viruses. Common colds are caused by several different viruses, and is present in the human population for many centuries, and re-emerge annually as seasonal epidemics and there is no evidence for any worsening in its pathogenicity/virulence. We can only sincerely hope that COVID-19 will also follows the same fate, but virus lingering is the cause of concern. Countries have to collaborate and not compete on a global vaccination campaign.

Some questions arise: How to control the progression of mutations? It is still not clear whether Covid-19 survival creates immunity to future infection and that too for how long? By now it is clear that as time goes on and the virus spreads, virus gets more time to evolve. So, massive scale vaccination campaign worldwide is to be undertaken and to be completed in a very short time. This can control the number of antigenic drift (variants) and antigenic shift (new viruses). Can one make a trade-off between virus spread by infection and vaccination to reach the HIT value very quickly?

\section{A new dawn in the fight against the disease: to be or not to be worried?}

Covid 19 cases are rising in certain countries as shown in fig. 2. What is the cause of this rise? Is it seasonal or new variants? If seasonal then one has to tackle it like Flu and if it is due to some other reason causing infection by new variant then it is a point of concern. Above we have discussed advantages of vaccination and the negative aspect of mutation leading to antigenic drift and shift causing immunity escape.

Another concern is how long the immunity last after natural infection and vaccination. If the immunity wanes, then there would be frequent reinfections resulting in severe symptoms and the virus will bounce back even in places that have reached the herd immunity threshold value. This means we are not going to be done with it in any time soon. Thus, the prior exposure or vaccination doesn't lessen your chance of winding up in the hospital. Recently several severe reinfections have been reported worldwide but still not reported on a larger scale. To overcome this, we need a vaccine that blocks corona virus transmission as was discussed above (sterilizing vaccine which clear the virus from your system entirely as in smallpox vaccine). A lack of sterilizing immunity means that the pathogen can still continue to circulate in the population, infecting unvaccinated and vulnerable and may even evolve to evade our immune responses.

\section{Judging the risk- "risk management":}

For many, the risk of dying from other illnesses and diseases like cancer etc., and accidents are the biggest cause of fatalities and is of greater concern than dying of coronavirus infection. Higher risks are for over 70 years of age and with poor health conditions ranging from diabetes to heart conditions, severe respiratory disease and immune compromised individuals. There will never be a "no risk situation", if the Covid-19 is present in the community. It is about how we can reduce the risk, just as we do with other kinds of daily dangers like smoking, alcohol consumption, driving, etc., COVID-19 pandemic should not spark nosophobia in individuals and community. One should not be bogged down thinking about the worst-case scenario because health-related anxiety or worry can become extremely uncomfortable and one can miss all the wonders of life. The vast majority of cases are going to be mild, and people are going to recover just like they do from a cold or flulike illness. Coronavirus is believed to be very contagious when patients are symptomatic, if one suspects to be infected then it is better to isolate themselves until their cough, fever and other symptoms go away to avoid spreading the virus. Shortness of breath, an unremitting fever or feeling extremely weak will surely require medical attention.

\section{Prophylactic measures for covid 19: Changing Diet and life style.}

Apart from therapeutic and vaccine intervention we all know from our childhood a saying that "prevention is better than cure", it is easier to be cautious than repairing the damage. The most popular preventing measure is to improve our natural (innate) immunity. 
Our immune system protects us from pathogenic infection (such as bacteria, viruses, fungi and parasites) as discussed above in the natural immunity section. Our natural immune system is always active carrying out non-stop surveillance with specialized cell and communicating molecules to deal with the threats if encountered. This activity is enhanced upon infection, metabolism rate and energy sources requirement increase along with requirement of substrates for biosynthesis and regulatory molecules, which are all ultimately derived from the diet. A number of vitamins (such as A, B6, B12, folate, $\mathrm{C}, \mathrm{D}$ and $\mathrm{E}$ etc.,) and trace elements (such as zinc, copper, selenium, iron, etc.,), amino acids and fatty acids are all required in supporting the immune system to deal with the invader (infection). Dietary correction may be required to achieve a healthy immune system. Severe infection of the respiratory epithelium can lead to acute respiratory distress syndrome (ARDS), characterized by excessive and damaging host inflammation, termed a cytokine storm which can be controlled by $n-3$ fatty acids [33]. Hence an adequate supply of a wide range of nutrients is essential to support the immune system to function optimally. Changing life style apart from diet such as exercise, yoga, meditation, intermittent fasting, proper sleeping, quitting drug addiction all these factors improve the innate immunity. Thus, other than therapeutic and vaccine prophylactic measures which may in rare cases have very severe harmful side effect on the other hand changing diet and life style will improve your body, mind and immune system.

\section{Maneuvering the pandemic:}

Now we have understood the intricacies involved in this pandemic. Now we can ask few questions. To answer them first let us recapitulate the facts gathered so far. Some facts obtained so far:

1. There are already several variants of SARS-CoV-2 detected by genomic surveillance.

2. These new variants are highly transmissible compared to wild type.

3. As the virus lingers and as time goes on, the virus spreads, virus gets more time to evolve due to selection pressure giving rise to variants (antigenic drift).

4. Antigenic drift favors RBD: ACE2 binding leading to higher transmission.

5. Leaky imperfect vaccine favors virus to overcome host immunity.

6. Variant prepares to jump from old population with weak immunity (old breeding ground) to younger population (new breeding ground) [34,35].

7. Mutated forms of SARS-CoV-2 able to reinfect people who have recovered from an earlier infection [36].

8. Vaccinated population can still spread virus $[37,38]$
Now we would like to pose few questions step by step and slowly let us try to comment on them too simultaneously:

Q1. When, how and will the herd immunity be achieved?

Comment. When the virus comes to term with immunity, herd immunity will slowly set in even if the virus is "leaky". Remember we have seen above that any intervention increases the immune pressure on the virus and will inevitably lead to selective viral immune escape. We cannot come to terms with the virus "surrendering to virus", may cause catastrophic loss of human lives. But Christie Aschwanden argues why herd immunity for covid is probably impossible (see reference 38)

Q2. Consequence of mass vaccination campaigns with vaccine not properly match with the $\mathrm{S}$ protein of highly infectious variants.

Comment. Use of these vaccines may give rise to more infectious immune escape variants as discussed above. It will be important to develop interventions (vaccine) capable of preventing transmission of diverse SARS-CoV-2 variants.

Q3. The natural antibodies produced initially in response to most antigens are high molecular weight immunoglobulins (IgM) with low affinity for the antigen (but have high avidity for the virus surface), while the antibodies produced by vaccine (e.g., IgG and IgA) have on average, orders of magnitude higher affinity for that antigen and hence will this hamper binding of natural antibodies to RBD of SARS-CoV-2 variant? What is the role of "Original antigenic sin" on SARS-CoV-2, requirement of improving vaccines and vaccination strategies?

Comment. If this is so i.e., suppression of binding of natural antibodies (IgM, variantnonspecific) in the presence of vaccine induced antibodies ( $\operatorname{IgG}$ and $\operatorname{IgA}$ ) then the first line protection is hampered to fight against Infection caused by highly infectious variants. Thus, it may happen that vaccination of young and healthy people will lead to suppression of their variant-nonspecific innate immune defense without efficiently activating the targeted adaptive immune response. This may delay the recovery of the disease/infection. This prolonged infection will lead to symptomatic infection and shedding of infectious variants.

It is interesting to note that this hampering of natural immunity is related to a very well-known phenomenon "Original antigenic sin", which states simply that prior exposure to an antigen leads to a subsequent suboptimal immune response to a related antigen [39-41]. Original antigenic sin could make vaccinated people more susceptible to the virus than those who are unvaccinated [42]. It was also observed in the case of Influenza that sequential influenza vaccination might lead to reduced vaccine 
effectiveness (VE). Recent observations have provided convincing evidence that reduced VE after sequential influenza vaccination is a real phenomenon and such reduction in VE be termed "negative antigenic interaction," [43]. This demands us to support development of improved SARS-CoV-2 vaccines and vaccination strategies.

Since this question appears to be more important to be addressed and interesting, let us understand it a little bit more on the concept of "Original Antigenic Sin", in the context of viral infections. "Original Antigenic Sin" implies that when the epitope varies slightly, then the immune system relies on memory of the earlier infection, rather than mount another primary or secondary response to the new epitope. Instead of adapting, the response relies on its memory of previous infection to mount an attack (response) and this response may be inadequate against the new strain. So, in the case of vaccines, if we only neutralize a single strain or epitope, and if that strain/epitope changes over time, then the immune system will be unable to mount a targeted secondary response. The disease enhances and lead to induce anergy (anergy means immune system is unable to mount a normal immune response against a specific antigen). This triggers loss of pathogen control and inducing abnormal clinical consequences. (For more detail on this see reference [44])

Q4. Is it likely that mass vaccination will result into viral resistance to the vaccines?

Comment: If we are convinced that more variants are likely to appear due to mutations which are very common in RNA viruses as they keep making mistakes in copying (no error correction). The variants which are talked about now are B.1.1.7 (first detected in the UK), B.1.351 (first detected in South Africa), and P.1 (detected in Manaus, Brazil) due to selection/immune pressure as mentioned earlier. Then by applying the phenomenon of "Original Antigenic Sin" as observed in the case of influenza vaccine, SARS-CoV-2 vaccine will ultimately have to follow like we are following for the case of influenza vaccine. Thus, vaccine-induced immune responses will inevitably result in a dramatic enhancement of infection rates in all of the vaccinated population when exposed to highly infectious Covid-19 variants.

Q5. Will large scale vaccination campaigns along with containment measures expedite antigenic drift causing more highly infectious Covid-19 variants?

Comment: This is one of the most important question and from the discussion above in this article this cannot be ruled out. Several references are mentioned in this article and they point in this direction.

Q6. Can Covid-19 vaccines generate herd immunity?

Comment: $\quad$ From the discussions in this article, it is very clear that shedding and transmission of highly infectious variants due to imperfect vaccine (imperfect vaccinal antibodies) will increasingly turn the vaccine recipients into asymptomatic virus spreaders. This will comprise a large source for infecting non-immunized portion of the population. It is also believed that during the natural course of pandemic the mass vaccination campaigns will never succeed in generating sufficient herd immunity to bring the pandemic under control, which we have seen in the case of domestic life stock or animal breeding farms $[44,45]$. Establishing herd immunity is made more difficult by presence of variants, varied host response to vaccination and also vaccines which provide only partial protection for a limited period of time (see reference 37 ).

Q7. Can individuals train the non-specific innate immunity by regular exposure to SARS-CoV-2 or other similar pathogens and promote the immunity by a healthy lifestyle.

Comment: This strategy has to be adopted and may be this will be our strong and only weapon left to fight SAR-CoV-2 unless more updated broad-spectrum vaccine is produced.

\section{Where are we now: A birds eye view}

For the sake of completeness, we would now like to point out some latest studies related to cross immunity, severity of delta variant prevailing in the world presently and natural immunity versus vaccine immunity. It has been pointed out recently [46] that antibodies produced against highly prevalent common seasonal coronaviruses ( $\mathrm{sCoVs}$ ) which causes common cold cross-react with SARS-CoV-2 antigens. This cross-reactivity plays a protective role against SARSCoV-2 infections and COVID-19 disease severity [46]. Galipeau et al. [46] showed that exposure to sCoVs triggers various cellular and humoral immune responses (antibody-mediated immunity) that influence the efficiency of SARS-CoV-2 spike binding to ACE2 and hence impact COVID-19 disease severity. Also, recently many other studies have demonstrated evidences for antibody and T cell crossneutralization causing protection against COVID-19 disease severity [47-53]. Humoral immune response plays an important role in clearing viral infection and memory response which helps to prevent virus reinfection [54]. Result obtained in Rhesus macaques showed that immune response generated after first SARS-CoV-2 infection protects against reinfection [55].

Interestingly recent studies on SARS-CoV-2 infection revealed that neutralizing antibodies correlate with disease severity and observed to be almost undetectable in patients with mild or asymptomatic infection [56,57]. Similar observation was found for the case of SARS-CoV-1 infection where severe pulmonary disease was higher in those subjects showing higher levels of neutralizing antibody titers [58]. For common coronaviruses (sCoVs) infection it was observed that specific IgG and neutralizing 
antibodies disappear within 1 year [59], while for SARS-CoV-1 and MERS-CoV antibody responses wane in a few years with a partial protection from reinfection [60]. Thus, question arises whether humoral [61] and cell mediated [62-64] immune response with cross-reactivity might be sufficient for cross-protection [52] and thus avoiding severe disease [61].

It has also been reported $[47,65]$ that children who had never been exposed to SARS-CoV-2 has higher levels of cross-reactive antibodies to the spike glycoprotein subunit S2 of SARS-CoV-2 than adults, indicating that children may have a higher degree of pre-existing cross-reactive immunity that provides them some level of protection from severe COVID-19. Apart from humoral cross-reactive immunity to SARS$\mathrm{CoV}-2$, it has also been shown that high frequencies of uninfected individuals mount pre-existing crossreactive $\mathrm{T}$ cell immune responses to SARS-CoV-2 [52,65 - 69].

It has been observed that antibody levels peak and then declines thereafter with time, while antibody avidity increases with time which suggest antibody maturation [70]. Immune responses were found to vary greatly among vaccinees and vaccines too [70]. Personalized long-time monitoring of antibodies may be necessary to assess the immunity duration of vaccinated individuals. It is therefore important to monitor immune responses both at the individual and population level and identify those who are still at risk even after vaccination and provide measures to protect them from infections [71,72].

In the current SARS-CoV-2 a variety of mutations have been accumulated in the viral genome and the newly emerged variants of concerns (VOCs) is the B.1.617.2 - Delta variant associates with a huge COVID-19 surge in India in Spring 2021. It has been reported [73] that the B.1.617.2 - Delta variant is highly fusogenic and more pathogenic than prototypic SARSCoV-2 in infected hamsters.

It has been reported [74] that memory antibodies selected over time by natural infection have greater potency and longevity than antibodies elicited by vaccination, hence suggesting that booster vaccination with currently available mRNA vaccines would produce a quantitative increase in plasma neutralizing activity but may not have much qualitative advantage against variants.

Assessing the duration of humoral and cellular immunity remains key to overcome the current SARS-CoV-2 pandemic, it has been reported that protective immune response is maintained up to nine months after SARS-CoV-2 infection in the absence of re-infection [75]. But interestingly it has also been reported that in the study [76] on the reactivity of antibodies with the $S$ protein of the original strain Wuhan-1 and the variant B.1.1.7 (Alpha) was found to have a significant difference in the titer of anti-S antibodies produced after a first dose of the vaccines
ChAdOx1 nCov-19/AstraZeneca, mRNA1273/Moderna, BNT162b2 /Pfizer-BioNTech and Ad26.COV.S/Janssen. It was found that there is a relative reduction in the reactivity of the sera with the B.1.1.7 versus the Wuhan- variant after the second boosting immunization. This cast doubts about the convenience of repeatedly immunizing with the same S protein sequence. It has also been reported [77] that convalescent subjects previously infected with ancestral variant SARS-CoV-2 produce antibodies that cross-neutralize emerging VOCs with high potency. It is now believed that after recovery from COVID-19 the viral-specific protective immunity may last over 1 year [78]

\section{Remarks - Take away message:}

Either our immunity has to come to terms with the virus or virus have to come to terms with our immunity. We believe good sense has to prevail. Instead of focusing the target we have to focus on the path. May be changing of gear is required to reach closer to the desired target even if the ultimate target is not achievable.

\section{Conclusion:}

Now it is time to collect all information and facts and look for similarity with other viral diseases which have been pointed in this article. We have seen confirmation of several variants; we have seen several causes for this variant to appear. We see that this virus is on the move from older population to younger population. The virus is becoming more virulent and more infectious immune escape variants. Updated broad-spectrum vaccine is required until then we have to promote the immunity by a healthy lifestyle and train the non-specific innate immunity and this will be our strong weapon to fight SAR-CoV-2.

\section{${ }^{*}$ Ex-Senior Professor, SINP, Kolkata}

Ethical approval: This study did not require ethical approval

Conflict of interest: None

Declarations of interest: None

\section{References:}

[1]. Andrew F. Read, et al., Imperfect vaccination can enhance the transmission of highly virulent pathogens. PLoS Biol 13(7): e1002198. (2015).

[2] Margaret J. Mackinnon and Andrew F. Read, Immunity promotes virulence evolution in a malaria model. PLoS Biol 2: E230 (2004).

[3] Victoria C. Barclay, et. al., The evolutionary consequences of blood-stage vaccination on the rodent malaria Plasmodium chabaudi. PLoS Biol 10: e1001368 (2012). 
[4] Gandon S, Mackinnon MJ, Nee S, Read AF, Imperfect vaccines and the evolution of pathogen virulence. Nature 414: 751-756. (2001).

[5] Mike Boots, The need for evolutionarily rational disease interventions: Vaccination can select for higher virulence. PLoS Biol 13:e1002236. (2015).

[6] Fleming-Davies et al., Incomplete host immunity favors the evolution of virulence in an emergent pathogen. Science 359, 1030-1033 (2018).

[7] Mackinnon MJ, Gandon S, Read AF Virulence evolution in response to vaccination: The case of malaria. Vaccine 26: C42-C52. (2008).

[8] P. D. Williams, T. Day, Epidemiological and evolutionary consequences of targeted vaccination. Mol. Ecol. 17, 485-499 (2008).

[9] M. G. M. Gomes, A. Margheri, G. F. Medley, C. Rebelo, Dynamical behaviour of epidemiological models with sub-optimal immunity and nonlinear incidence. J. Math. Biol. 51, 414-430 (2005).

[10] W. M. Geisler, S. Y. Lensing, C. G. Press, E. W. Hook 3rd, Spontaneous Resolution of Genital Chlamydia trachomatis Infection in Women and Protection from Reinfection. J. Infect. Dis. 207, 1850 1856 (2013).

[11] S. Hall et al., Second Varicella Infections: Are They More Common Than Previously Thought? Pediatrics 109, 1068-1073 (2002).

[12] X. Castellsagué et al., Risk of Newly Detected Infections and Cervical Abnormalities in Women Seropositive for Naturally Acquired Human Papillomavirus Type 16/18 Antibodies: Analysis of the Control Arm of PATRICIA. J. Infect. Dis. 210, 517534 (2014).

[13] R. Casais et al., Primary and secondary experimental infestation of rabbits (Oryctolagus cuniculus) with Sarcoptes scabiei from a wild rabbit: factors determining resistance to reinfestation. Vet. Parasitol. 203, 173-183 (2014).

[14] M. C. De Jong, et. al., Quantitative investigation of population persistence and recurrent outbreaks of bovine respiratory syncytial virus on dairy farms. Am. J. Vet. Res. 57, 628-633 (1996).

[15] A. Sabó, D. Blaškovic, Resistance of pig tonsillary and throat mucosa to reinfection with a homologous pseudorabies virus strain. Acta Virol. 14, 17-24 (1970).

[16] J. L. Schulman, E. D. Kilbourne, Induction of partial specific heterotypic immunity in mice by a single infection with influenza a virus. J. Bacteriol. 89(1), 170-174 (1965).

[17] Read AF, Mackinnon MJ, Pathogen evolution in a vaccinated world. In: Stearns SC, Koella J, editors.
Evolution in Health and Disease 2nd ed. Oxford: Oxford University Press. pp. 139-152. (2008).

[18] Kevin R. McCarthy et al., Recurrent deletions in the SARS-CoV-2 spike glycoprotein drive antibody escape, Science 371, 1139-1142 (2021).

[19] Emily Shang, Paul H. Axelsen, The Potential for SARS-CoV-2 to Evade Both Natural and Vaccineinduced Immunity, bioRxiv preprint doi: 10.1101/2020.12.13.422567; December 13, (2020).

[20] Hansen et al., Studies in humanized mice and convalescent humans yield a SARS-CoV-2 antibody cocktail. Science 369, 1010-1014 (2020)

[21] Baum et al., Science 369, 1014-1018 (2020), Antibody cocktail to SARS-CoV-2 spike protein prevents rapid mutational escape seen with individual antibodies.

[22] Baum et al., REGN-COV2 antibodies prevent and treat SARS-CoV-2 infection in rhesus macaques and hamsters. Science 370, 1110-1115 (2020),

[23] B. Agerer et al., SARS-CoV-2 mutations in MHCI-restricted epitopes evade CD8+ T cell responses. Sci. Immunol. 10.1126/sciimmunol.abg6461 (2021).

[24] Weisblum, Schmidt, et al., Escape from neutralizing antibodies by SARS-CoV-2 spike protein variants. eLife 2020;9:e61312. DOI: https://doi.org/10.7554/eLife.61312,

[25] Greaney et al., Complete Mapping of Mutations to the SARS-CoV-2 Spike Receptor-Binding Domain that Escape Antibody Recognition. Cell Host \& Microbe 29, 44-57 January 13, (2021)

[26] Ewen Callaway, "Making sense of coronavirus mutations" Nature | Vol 585 | 10 September 2020

[27] Korber et al., Tracking Changes in SARS-CoV-2 Spike: Evidence that D614G Increases Infectivity of the COVID-19 Virus. Cell 182, 812-827 August 20, (2020),

[28] Cody B. Jackson et. al., Functional importance of the D614G mutation in the SARS-CoV-2 spike protein. Biochemical and Biophysical Research Communications 538108 - 115, (2021)

[29] Kai Kupferschmidt, "Vaccine makers ponder how to adapt to virus variants" 29 January, vol 371 issue 6528 pg 448. (2021)

[30] Elisabeth Mahase, Covid-19: Where are we on vaccines and variants? BMJ 2021;372:n597 http://dx.doi.org/10.1136/bmj.n597 Published: 02 March (2021)

[31] Ewen Callaway \& Heidi Ledford, How to redesign COVID vaccines so they protect against variants. Nature 590, 15-16 (2021) doi: https://doi.org/10.1038/d41586-021-00241-6 
[32] W. F. Garcia-Beltran et al., Multiple SARS-CoV2 variants escape neutralization by vaccine-induced humoral immunity. Cell 184, 1-12 April 29, (2021),

[33] Calder PC. Nutrition, immunity and COVID-19. BMJ Nutrition, Prevention \& Health 2020;0. doi:10.1136/ bmjnph-2020-000085

[33] Sarah Brookman, et. al., Effect of the new SARSCoV-2 variant B.1.1.7 on children and young people, Lancet, www.thelancet.com/child-adolescent Vol 5 pg. e9, April (2021)

[34] Luke Taylor, Covid-19: Brazil's spiraling crisis is increasingly affecting young people BMJ 2021;373:n879 doi:10.1136/bmj.n879 Published: 01 April (2021)

[35] Richard L Tillett et. al., "Genomic evidence for reinfection with SARS-CoV-2: a case study", Lancet Infect Dis 2020; 21: 52-58 Published Online October 12, (2020).

[36] Nicholas J. Savill et. al. "Silent spread of H5N1 in vaccinated poultry", NATURE, Vol 442, 17 August 2006 pg. 757

[37] Christie Aschwanden, "Why herd immunity for covidis probably impossible", Nature, Vol 591, 25 March 2021, 520.

[38] T Francis, Influenza: the new acquayantance. Ann Intern Med 39: 203-221 (1953).

[39] Fazekas de St Groth S, Webster RG, Disquisitions on original antigenic sin I. Evidence in man. J Exp Med 124: 331-345 (1966).

[40] Fazekas de St Groth S, Webster RG Disquisitions on original antigenic sin II. Proof in lower creatures. J Exp Med 124: 347-361 (1966).

[41] Susanne L. Linderman and Scott E. Hensley, Antibodies with 'Original Antigenic Sin' Properties Are Valuable Components of Secondary Immune Responses to Influenza Viruses, PLoS Pathog 12(8):e1005806. (2016).

[42] Arnold S. Monto, et. al., The Doctrine of Original Antigenic Sin: Separating Good from Evil. JID:215, 1784 (2017)

[43] Anup Vatti et. al. "Original antigenic sin: A comprehensive review", Journal of Autoimmunity 83 (2017) 12-21.

[44] K.E. Atkins et al. The effectiveness of mass vaccination on Marek's disease virus (MDV) outbreaks and detection within a broiler barn: A modelling study Epidemics 5 (2013) 208-217

[45] T. J. D. Knight-Jones et. al., Mass vaccination, immunity and coverage: modelling population protection against foot-and-mouth disease in Turkish cattle, Scientific Reports 6:22121 (2016) DOI: 10.1038/srep22121
[46] Yannick Galipeau et al., Relative Ratios of Human Seasonal Coronavirus Antibodies Predict the Efficiency of Cross-Neutralization of SARS-CoV-2 Spike Binding to ACE2, medRxiv preprint doi: https://doi.org/10.1101/2021.07.16.21260079; 18, 2021

[47] K. W. Ng et al., Preexisting and de novo humoral immunity to SARS-CoV-2 in humans. Science 370, 1339-1343 (2020).

[48] J. Braun et al., SARS-CoV-2-reactive T cells in healthy donors and patients with COVID-19. Nature 587, 270-274 (2020).

[49] M. Sagar et al., Recent endemic coronavirus infection is associated with less-severe COVID-19. The Journal of Clinical Investigation 131, (2021).

[50] W. R. Morgenlander, et al., Antibody responses to endemic coronaviruses modulate COVID-19 convalescent plasma functionality. J Clin Invest 131, (2021).

[51] I. Schulien, et al., Characterization of pre-existing and induced SARS-CoV-2- specific CD8(+) T cells. Nat Med 27, 78-85 (2021).

[52] J. Mateus et al., Selective and cross-reactive SARS-CoV-2 T cell epitopes in unexposed humans. Science 370, 89-94 (2020).

[53] Heffron AS, et al. The landscape of antibody binding in SARS-CoV-2 infection. PLoS Biol 19(6): e3001265 (2021)

[54] L. Guo et al., Cross-reactive antibody against human coronavirus OC43 spike protein correlates with disease severity in COVID-19 patients: a retrospective study, Emerging Microbes \& Infections, 10664 (2021)

[55] Chandrashekar A, et al., SARS-CoV-2 infection protects against rechallenge in rhesus macaques. Science 369:812-7 (2020).

[56] E. Percivalle et al. Prevalence of SARS-CoV-2 specific neutralising antibodies in blood donors from the Lodi red zone in Lombardy, Italy, Euro Surveill;25:2001031 (2020).

[57] Long QX, et al. Antibody responses to SARSCoV-2 in patients with COVID-19. Nat Med; 26:8458 (2020). Carlo Cervia et al., Systemic and mucosal antibody secretion specific to SARS-CoV-2 during mild versus severe COVID-19, bioRxiv preprint doi: https://doi.org/10.1101/2020.05.21.108308; May 23, 2020

[58] L. Zhang, et al. Antibody responses against SARS coronavirus are correlated with disease outcome of infected individuals. J Med Virol 78:1-8. (2006). 
[59] Callow KA, Parry HF, Sergeant M, Tyrrell DA. The time course of the immune response to experimental coronavirus infection of man. Epidemiol Infect; 105:435-46 (1990).

[60] Yang L, Peng H, Zhu Z, Li G, Huang Z, Zhao Z, et al. Persistent memory CD4+ and CD8+ T-cell responses in recovered severe acute respiratory syndrome (SARS) patients to SARS coronavirus M antigen. J Gen Virol; 88:2740-8 (2007).

[61] Zhao Q, He Y. Challenges of convalescent plasma therapy on COVID-19. J Clin Virol; 127:104358 (2020).

[62] Weiskopf D, Schmitz KS, Raadsen MP, Grifoni A, Okba NMA, Endeman H, et al. Phenotype and kinetics of SARS-CoV-2-specific T cells in COVID-19 patients with acute respiratory distress syndrome. Sci Immunol;5:eabd2071 (2020).

[63] Braun J, Loyal L, Frentsch M, Wendisch D, Georg $\mathrm{P}$, Kurth F, et al. SARS-CoV-2-reactive $\mathrm{T}$ cells in healthy donors and patients with COVID-19. Nature, 587:270-4 (2020).

[64] Mathew D, Giles JR, Baxter AE, Oldridge DA, Greenplate AR, Wu JE, et al. Deep immune profiling of COVID-19 patients reveals distinct immunotypes with therapeutic implications. Science;369:eabc8511 (2020).

[65] Elizabeth Fraley et al., Cross-reactive antibody immunity against SARS-CoV-2 in children and adults, Cellular \& Molecular Immunology 18:1826 - 1828 (2021).

[66] Shrock, E. et al. Viral epitope profiling of COVID19 patients reveals cross reactivity and correlates of severity. Science 370, https://doi.org/10.1126/science.abd4250 (2020).

[67] Grifoni, A. et al. Targets of T Cell Responses to SARS-CoV-2 Coronavirus in Humans with COVID-19 Disease and Unexposed Individuals. Cell 181, 14891501.e1415 (2020).

[68] Swapnil Mahajan et al., Immunodominant T-cell epitopes from the SARS-CoV-2 spike antigen reveal robust pre-existing T-cell immunity in unexposed individuals, Scientific Reports 11:13164 (2021).

[69] Irene Cassaniti et al., SARS-CoV-2 specific T-cell immunity in COVID-19 convalescent patients and unexposed controls measured by ex vivo ELISpot assay, Clinical Microbiology and Infection 27 10291034 (2021)

[70] Kevin P. Bliden et al., Evolution of Anti-SARSCoV-2 IgG Antibody and IgG Avidity Post Pfizer and Moderna mRNA Vaccinations, medRxiv preprint doi: https://doi.org/10.1101/2021.06.28.21259338; July 2, 2021.

[71] Elitza S. Theel et al., The Role of Antibody Testing for SARS-CoV-2: Is There One?, Journal of Clinical Microbiology 58 (8) e00797-20 (2020).

[72] Alexis R. Demonbreun et al., Comparison of IgG and neutralizing antibody responses after one or two doses of COVID-19 mRNA vaccine in previously infected and uninfected individuals, EClinicalMedicine 38101018 (2021).

[73] Akatsuki Saito et al., SARS-CoV-2 spike P681R mutation, a hallmark of the Delta variant, enhances viral fusogenicity and pathogenicity, bioRxiv preprint doi: https://doi.org/10.1101/2021.06.17.448820; July 19,2021

[74] Alice Cho et al., Antibody Evolution after S 1 ARS-CoV-2 mRNA Vaccination, bioRxiv preprint doi: https://doi.org/10.1101/2021.07.29.454333; July 29,2021

[75] Vo Hoa Thi My et al., Robust and Functional Immunity up to 9 months after SARS-CoV-2 infection: a Southeast Asian longitudinal cohort. bioRxiv preprint doi: $\quad$ https://doi.org/10.1101/2021.08.12.455901; August 12, 2021

[76] Lydia Horndler et al., Decreased Breadth Of The Antibody Response To The Spike Protein Of SarsCov-2 After Vaccination, medRxiv preprint doi: https://doi.org/10.1101/2021.08.12.21261952; August 14,2021

[77] L. Wang et al., Science 373, eabh1766 (2021). DOI: $10.1126 /$ science.abh1766

[78] Chengqian Feng et al., Protective humoral and cellular immune responses to SARS-CoV-2 persist up to 1 year after recovery, Nature Communications 12:4984 (2021) 